\title{
Characteristics of Eight Pediatric Patients with Coronavirus Disease
}

\section{9}

\author{
Gaoyan Chen ${ }^{1}$, Junhua $\mathrm{Li}^{1}$, Yongchao Jiang ${ }^{1}$, Haihua Chen ${ }^{1}$ and Rui Pan ${ }^{1, *}$ \\ ${ }^{1}$ Department of Pediatrics, Xiangyang Central Hospital, Affiliated Hospital of Hubei University of Arts and Science, Xiangyang City, China \\ "Corresponding author: Department of Pediatrics, Xiangyang Central Hospital, Affiliated Hospital of Hubei University of Arts and Science, No.136 Jinzhou Street, Xiangcheng \\ District Xiangyang City, 441021, China. Tel: +86-139-8639-4739, Email: prdoctor1979@163.com
}

Received 2020 April 04; Revised 2020 May 26; Accepted 2020 August 07.

\begin{abstract}
Background: Coronavirus Disease 2019 (COVID-19) has attracted global attention for unexpected rapid transmission among countries. Currently, little is known about the attributes of pediatric patients with COVID-19.

Objectives: To describe the characteristics of pediatric patients with COVID-19.

Methods: We retrospectively analyzed the epidemiological, laboratory, imaging, and clinical data of pediatric patients with COVID19 from January 31 to February 24, 2020.

Results: A total of eight patients with COVID-19 were enrolled in this study. Two patients had underlying diseases. Seven children (87.5\%) belonged to a family cluster outbreak. The most common symptoms were fever (50\%), cough (37.5\%), expectoration (37.5\%), and Sore throat (37.5\%). For the laboratory findings, all patients had positive nCoV-RNA results, three patients (37.5\%) with abnormal white blood cell counts, and six patients (75\%) with higher lymphocyte counts. Chest computed tomography (CT) showed groundglass opacity or patchy shadows in 7 patients (87.5\%). All patients received antiviral therapy. Additionally, four patients (50\%) were treated with antibiotics, one with glucocorticoid atomization (12.5\%), one with interferon atomization (12.5\%), four patients (50\%) with traditional Chinese medicine. All patients discharged within three weeks, without poor prognosis or recurrence after 14 days follow-up.

Conclusions: Pediatric patients with COVID-19 were mainly transfected through family cluster outbreaks. The clinical manifestations were mild without a typical pattern. High-resolution CT and nCoV-RNA tests have great value for early-stage diagnosis. Antiviral therapy is effective in treating viral pneumonia.
\end{abstract}

Keywords: Child, Infection, Coronavirus, Pneumonia, Treatment

\section{Background}

Currently, Coronavirus Disease 2019 (COVID-19) has attracted global attention for unexpected rapidly spread attributed to extensive traveling among countries. Until February 24, 77658 cases were confirmed in China within just two months, leading to 2663 deaths (1). Around 1630 (2.1\%) cases were children under ten years old (2). Compared to adults, pediatric patients were fewer and disease manifestation in them milder (3). Since many suspected patients were in quarantine, the actual number of infected cases may increase with widely used pathogen tests.

With the disease spreading, people pay more attention to COVID-19 and find that COVID-19 seems to have a lower fatality, but higher infectivity compared with the Middle East respiratory syndrome (MERS) and the severe acute respiratory syndrome (SARS) (4). Although only two severe pediatric cases were reported in China and no cases of death, we still need to be vigilant as children are a particular population. Children are susceptible to be infected with respiratory viruses due to the immature immune system and respiratory tract structure (5).

The spread of COVID-19 among children is mainly conducted by human-to-human in family settings (6). Their clinical symptoms may be diverse and atypical, even without symptoms, and the incubation period could be more than 14 days, all of these make the diagnosis more difficult (7). It is worth noting that since respiratory viruses are common during the fall and winter, children with COVID19 also could be infected with other viruses.

\section{Objectives}

Although there are many diagnosis and treatment recommendations, prevention and isolation remain the primary effective control measures. Since it is a new challenge 
never met before, we designed this study to summarize the characteristics of pediatric patients with COVID.

\section{Methods}

\subsection{Study Design and Participants}

This retrospective study was performed from January 17 to February 24, 2020, at Xiangyang Central Hospital. A total of 166 suspected child patients were admitted, and eight of these patients were diagnosed with laboratoryconfirmed COVID-19 by quantitative real-time reverse transcription-PCR using nasopharyngeal swab specimens. Three children had negative nCoV-RNA tests at first, the results became positive after 3 to 5 days follow-up. Eight patients with laboratory-confirmed SARS-CoV-2 were finally enrolled based on the "Diagnosis, Treatment, and Prevention of 2019 Novel Coronavirus Infection in Children: Experts' Consensus Statment" (8).

This study was approved by the Ethics Committee of Xiangyang Central Hospital. Written informed consent was not obtained as the study was retrospective.

\subsection{Data Collection}

The final data, containing epidemiological, clinical manifestation, laboratory indicators, radiological features, treatment and outcomes, were obtained from the electronic medical records. After admission, nucleic acid test and high-resolution CT pulmonary scanning were performed immediately. We collected clinical manifestations data of all suspected patients, including fever, fatigue, dry cough, expectoration, running nose, pharyngalgia, headache, and some gastrointestinal symptoms such as abdominal discomfort, abdominal pain, nausea, vomiting, diarrhea. Laboratory examinations were obtained, including blood routine, blood clotting index, hepatorenal function, myocardial enzyme, procalcitonin (PCT), C reactive protein (CRP), erythrocyte sedimentation rate (ESR), and other pathogens as influenza B, mycoplasma pneumoniae, chlamydia pneumoniae. Typical CT findings were defined as interstitial changes and multiple small plaques in the lung periphery at early-stage, with further deteriorated to bilateral multiple ground-glass opacities or infiltrating shadows. Lung consolidation was observed in severe cases; pleural effusion was rare. The normal range of white blood cell (WBC) counts was $5-10 \times 10^{9} / \mathrm{L}$ while lymphocyte counts were $1.1-3.2 \times 10^{9} / \mathrm{L}$. Suspected cases were defined as those who had an epidemiological history and two of the following clinical manifestations: (a) Fever, fatigue, dry cough; (b) chest CT findings as mentioned above; (c) in the early stage, WBC count was normal or decreased, or lymphocyte count decreased; (d) no other pathogens were detected to explain the clinical manifestations. Confirmed cases were defined as suspected cases with 2019-nCoV nucleic acid positive. The release and discharge criteria included: (a) normal body temperature for more than three days in a row; (b) obvious improvement of the respiratory symptoms; (c) negative nCoV-RNA finding in two consecutive tests with an interval of at least one day (9).

\section{Results}

All patients aged from 1 to 14 years (Table 1$)$. Five were boys, and three were girls. Two patients (25\%) had underlying diseases: congenital ventricular septal defect in case 1 and cough variant asthma in case 2 . Seven children (87.5\%) associated with a family cluster outbreak. According to clinical manifestation, they were categorized into 3 types: 1 common case (case 3,12.5\%), 4 mild cases (cases 1, 2, 4 and 5, 50\%), and 3 asymptomatic cases (cases 6 - 8, 37.5\%). Fever was observed in cases 2 - 5 (50\%); cough, expectoration and sore throat were seen in cases 1-3(37.5\%). Case 4 also had an occasional mild headache. Their breathing rate, heart rate, blood pressure, and blood oxygen saturation were stable.

Besides COVID, two patients were infected with other pathogens: Case 3 (12.5\%) combined with influenza B; case 2 (12.5\%) combined with mycoplasma pneumoniae and chlamydia pneumoniae (Table 1 ). WBC counts of 5 patients were normal (62.5\%); only case 7 (12.5\%) had more than 10 $\times 10^{9} / \mathrm{L}$; in cases 3 and $4(25 \%)$ it as less than $4 \times 10^{9} / \mathrm{L}$. Neutrophils counts of 6 cases (cases 1 to 4,6 , and 8, 75\%) decreased. Lymphocyte counts of 6 cases (cases 1 to 4,6 , and $8,75 \%$ ) and monocyte counts of 4 cases (cases 1, 3, 6, and $8,50 \%$ ) increased. Two patients (cases 4 to $5,25 \%$ ) had mild increased D-dimer, three patients (cases 1, 2 and 5, 37.5\%) had increased serum lactate dehydrogenase, and six patients (cases 1 to 5 , and case $8,75 \%$ ) had decreased serum creatinine. However, other biochemical items were normal, including PCT, CRP, and ESR.

Every patient had received chest CT scanning after admission. The first CT imaging of 7 cases (87.5\%) showed pneumonia except case 6 (Table 1 ), whose follow-up CT images after three days showed virus pneumonia. Follow-up CT scans indicated their pneumonia relieved.

All patients were given antiviral treatment (lopinavir/ritonavir oral solution) (Table 1). Case 3 also took oseltamivir phosphate granules for the disease being combined with influenza $B$ and received intermittent oxygen inhalation due to unstable blood oxygen. Four cases $(50 \%)$ received antibiotic treatment (azithromycin) as combined with mycoplasma pneumoniae and chlamydia pneumonia. Four patients (cases 2, 3, 4 and 8) also received traditional Chinese medicine (TCM), which was 


\begin{tabular}{|c|c|c|c|c|c|c|c|c|}
\hline Items & Case 1 & Case 2 & Case 3 & Case 4 & Case 5 & Case 6 & Case 7 & Case 8 \\
\hline Age (year) & 2.5 & 7 & 8 & 9 & 1 & 7 & 14 & 11 \\
\hline Sex & boy & girl & girl & boy & boy & girl & boy & boy \\
\hline Exposure history & + & + & + & + & - & + & + & + \\
\hline Comorbidities & + & + & - & - & - & - & - & - \\
\hline \multicolumn{9}{|l|}{ Signs and symptoms } \\
\hline Fever & - & + & + & + & + & - & - & - \\
\hline Cough & + & + & + & - & - & - & - & - \\
\hline Expectoration & + & + & + & - & - & - & - & - \\
\hline Pharyngalgia & + & + & + & - & - & - & - & - \\
\hline Headache & - & - & - & + & - & - & - & - \\
\hline \multicolumn{9}{|l|}{ Pathogens } \\
\hline nCoV-RNA & $\mathrm{a}^{\mathrm{a}}$ & $\AA^{a}$ & + & + & + & + & $\mathrm{a}^{\mathrm{a}}$ & + \\
\hline Influenza B & - & - & + & - & - & - & - & - \\
\hline Mycoplasma pneumonia & - & + & - & - & - & - & - & - \\
\hline Chlamydia pneumonia & - & + & - & - & - & - & - & - \\
\hline Chest CT scan & $++^{\mathrm{b}}$ & $+^{\mathrm{c}}$ & $++^{\mathrm{b}}$ & $+{ }^{\mathrm{d}}$ & $++^{\mathrm{b}}$ & - & $+^{e}$ & $+^{c}$ \\
\hline \multicolumn{9}{|l|}{ Laboratory results } \\
\hline $\mathrm{WBC}(\times \mathrm{G} / \mathrm{L})$ & 7.19 & 5.36 & 3.36 & 2.32 & 6.35 & 4.15 & 12.07 & 5.40 \\
\hline Neutrophil (× G/L) & 19.20 & 37.40 & 32.70 & 32 & 4.32 & 41.70 & 45.70 & 37 \\
\hline Lymphocyte (× G/L) & 65.60 & 50.70 & 53 & 60 & 3.41 & 44.10 & 47.60 & 43.70 \\
\hline Monocyte $(\times \mathrm{G} / \mathrm{L})$ & 10.20 & 5.60 & 12.50 & 8 & 7.13 & 10.80 & 45.40 & 10.20 \\
\hline D-dimer (mg/L) & 0.38 & NA & 0.26 & 6.28 & 0.63 & 0.32 & NA & 0.14 \\
\hline $\mathrm{CK}(\mathrm{U} / \mathrm{L})$ & 222 & 130 & NA & 73 & 119 & 70 & 72 & 45 \\
\hline CK-MB (U/L) & 19 & 8 & NA & 27 & 21 & 8 & 5 & 16 \\
\hline $\mathrm{LDH}(\mathrm{U} / \mathrm{L})$ & 395 & 269 & NA & 196 & 338 & 236 & 153 & 208 \\
\hline $\operatorname{ALT}(\mathrm{U} / \mathrm{L})$ & 14 & 11 & 11 & 14 & 9 & 23 & 29 & 13 \\
\hline $\operatorname{AST}(\mathrm{U} / \mathrm{L})$ & 50 & 26 & 22 & 25 & 37 & 28 & 16 & 22 \\
\hline $\mathrm{UN}(\mathrm{mmol} / \mathrm{L})$ & 4.40 & 4.0 & 3.00 & 2.70 & 3.90 & 6.20 & 3.90 & 4.90 \\
\hline $\operatorname{Scr}(\mu \mathrm{mol} / \mathrm{L})$ & 20.20 & 36.90 & 28.80 & 28.70 & 27.80 & 32.90 & 42.80 & 53.70 \\
\hline PCT (ng/mL) & 0.03 & NA & NA & NA & 0.13 & 0.04 & NA & 0.03 \\
\hline $\mathrm{CRP}(\mathrm{mg} / \mathrm{L})$ & 5 & 3.02 & 4.49 & 5.01 & 5 & NA & - & - \\
\hline \multicolumn{9}{|l|}{ Treatment } \\
\hline Oxygen therapy & - & - & + & - & - & - & - & - \\
\hline Antiviral therapy & + & + & + & + & + & + & + & + \\
\hline Antibiotic treatment & + & + & - & - & + & - & + & - \\
\hline Glucocorticoid therapy & - & + & - & - & - & - & - & - \\
\hline Interferon therapy & + & - & - & - & - & - & - & - \\
\hline TCM & - & + & + & + & - & - & - & + \\
\hline Days of hospital stay (d) & 14 & 7 & 17 & 17 & 11 & 14 & 13 & 19 \\
\hline Duration of illness (d) & 18 & 11 & 18 & 18 & 14 & 15 & 16 & 20 \\
\hline Clinical outcome & $\mathrm{D}$ & $\mathrm{D}$ & D & D & D & D & D & D \\
\hline \multicolumn{9}{|c|}{$\begin{array}{l}\text { Abbreviations: ALT, alanine aminotransferase; AST, aspartate aminotransferase; CK, creatine kinase; Cr } \\
\text { lactate dehydrogenase; NA, not available; PCT, procalcitonin; Scr, serum creatinine; TCM, traditional c } \\
\text { a } \text { Three children had negative nCoV-RNA tests at first, then had positive results after } 3 \text { to } 5 \text { days follow- } \\
\text { b Scattered patchy ground-glass opacities in both lungs } \\
\text { c Patchy lesions in the upper lobe of the right lung } \\
{ }^{d} \text { Multiply ground-glass opacities and patchy lesions in the middle and lower lobes of the right lung } \\
\text { e Ground-glass opacity in the lower lobe of the left lung }\end{array}$} \\
\hline
\end{tabular}

made from glycyrrhiza, bitter almond, ephedra, gypsum, amomum, reed root, and trichosanthes.

According to the criteria (9), all patients were dis- charged within three weeks. The follow-up was ended after two negative nucleic acid tests (one test per week). No poor prognosis or recurrence was found during the follow- 
up period.

\section{Discussion}

No case of death was reported among pediatric patients to date; only two children with severe novel corona virus pneumonia (NCP) are published. However, the risk of death remains to be taken into account since pediatric death cases were reported during the SARS and MERS epidemics $(10,11)$. The clinical features of children with COVID19 are variable. The primary presentations are respiratory symptoms such as fever, cough, and expectoration, while the gastrointestinal symptoms including nausea, vomiting, and diarrhea are uncommon (5). Three patients without overt symptoms were diagnosed with RNA test due to their family exposure. Since the parents of case 5 denied any exposure history, we consider he could be infected through aerosols. Aerosols are a suspension system of solid or liquid particles in air. The droplet nucleus containing novel coronavirus could hover in the air for a long time. Flushing toilets may also generate aerosols leading to diffusion (12). Since excretion can also produce aerosols, it is unlikely that NCP spread through the fecal-oral route, aerosols and breathing may serve as the main routes of transmission.

After admission, blood routine tests showed often normal WBC counts; case 7 had more than $10 \times 10^{9} / \mathrm{L}$, indicating an accompanying bacterial infection; cases 3 and 4 had less than $4 \times 10^{9} / \mathrm{L}$, which met the diagnostic criteria of COVD-19. Case 1, 2, 5 had slightly increased D-dimer and $\mathrm{LDH}$ levels while in other cases these were normal, high levels being features of severe cases (8). Due to the above results, blood routine test can provide the clue for mild virus infection in pediatric patients. Typical images of NCP showed single or multiple patchy or nodular groundglass shadows accompanied by septal thickening (13). Our research indicated that the most pulmonary CT findings were high-density shadows. The primary CT features included multiple ground-glass opacities (cases 1, 3, 4, 5, and 7) and patchy lesions (cases 4 and 8). Case 6 had a normal chest CT result on admission. Yet, the follow-up CT scan showed multiple ground-glass opacities mainly located in the subpleural area of both lungs after three days. Groundglass opacities were the most common CT features, with a ratio of $14 \%$ to $77 \%$ in recently published studies (14-16), which were consistent with our CT findings. However, the CT images of NCP were diverse and overlapped with other inflammatory conditions sometimes. For those patients with atypical imaging features, we need to identify NCP by combining clinical characteristics and nucleic acid test.

Although a variety of guides and experts' consensuses recommend lots of treatment options, there is no specific treatment for COVID-19. The key measures to control the disease are early diagnosis and isolation. In the early stage, the essential treatment for infected patients was antiviral therapy with supportive treatment. All treatments were based on the "Diagnosis, Treatment, and Prevention of 2019 Novel Coronavirus Infection in Children: Experts' Consensus Statment" (8), which also recommended TCM. TCM primarily functions to remove heat from the lungs to relieve cough, to clear heat and remove toxicity, and to increase immunity (17). The Chinese herbals we used mainly include glycyrrhiza, bitter almond, ephedra, etc. Case 1 and case 2 were treated with atomizing inhalation according to their corresponding respiratory symptoms. In China, all hospitals responsible for the treatment of COVID-19 patients are assigned by the government. In these hospitals, patients are treated in single rooms equipped with independent oxygen sources to minimize the risk that COVID19 may transmit through aerosol. Because of the particularities and limitations of pediatric patients, the safety and efficacy of nebulizer therapy for children need to be further confirmed. All patients were discharged within three weeks with a good prognosis, and no poor prognosis or recurrence was found after 14 days follow-up.

\subsection{Limitations}

This report, as a retrospective study, has its limitations. It is a single-center study with only 8 confirmed cases, which may have impact on the representativeness of the conclusions. Besides, many asymptomatic and mild patients maybe have not referred to the hospital. So, it was tough to recruit sufficient patients for our research. As for the present cases, we have done a lot of work to ensure the rigor of the research. All patients enrolled in our study were jointly diagnosed and treated by a multidisciplinary diagnosis and treatment team composed of respiratory medicine staff, infectious disease experts, emergency medical staff, and critical care medical staff. Therefore, our results are reliable. In future, we will conduct multicenter studies to analyze the characteristics of pediatric COVID-19 patients.

\subsection{Conclusions}

Pediatric patients with COVID-19 were mainly transfected through family cluster outbreaks. The common clinical manifestations were mild without a typical pattern. High-resolution CT and nCoV-RNA tests have great value for early-stage diagnosis.

\section{Footnotes}

Authors' Contribution: G.Y.C. and R.P. designed the experiments. J.H.L., Y.C.J., and H.H.C. carried out the experi- 
ments and calculations. G.Y.C. wrote and edited the paper. Conflict of Interests: None.

Ethical Approval: This study was approved by the Ethics Committee of Xiangyang Central Hospital.

Funding/Support: This work was supported by the National Natural Science Foundation of China [grant number 81703910].

Informed Consent: Written informed consent was not obtained as the study was retrospective.

\section{References}

1. National Health Commission of People's Republique of China. Daily briefing on novel coronavirus. 2020. Available from: http://en.nhc.gov. cn/2020-02/24/c_76819.htm.

2. Epidemiology Working Group for Ncip Epidemic Response; Chinese Center for Disease Control Prevention. [The epidemiological characteristics of an outbreak of 2019 novel coronavirus diseases (COVID19) in China]. Zhonghua Liu Xing Bing Xue Za Zhi. 2020;41(2):14551. Chinese. doi: 10.3760/cma.j.issn.0254-6450.2020.02.003. [PubMed 32064853].

3. Wang XF, Yuan J, Zheng YJ, Chen J, Bao YM, Wang YR, et al. [Retracted: Clinical and epidemiological characteristics of 34 children with 2019 novel coronavirus infection in Shenzhen]. Zhonghua Er Ke Za Zhi. 2020;58(0). Chinese. E008. doi: 10.3760/cma.j.issn.05781310.2020.0008. [PubMed: 32062875].

4. Swerdlow DL, Finelli L. Preparation for Possible Sustained Transmission of 2019 Novel Coronavirus: Lessons From Previous Epidemics. JAMA. 2020;323(12):1129-30. doi: 10.1001/jama.2020.1960. [PubMed: 32207807].

5. Shen KL, Yang YH. Diagnosis and treatment of 2019 novel coronavirus infection in children: a pressing issue. World J Pediatr. 2020;16(3):21921. doi: 10.1007/s12519-020-00344-6. [PubMed: 32026147]. [PubMed Central: PMC7091265].

6. Cai J, Xu J, Lin D, Yang Z, Xu L, Qu Z, et al. A Case Series of children with 2019 novel coronavirus infection: clinical and epidemiological features. Clin Infect Dis. 2020. doi: 10.1093/cid/ciaa198. [PubMed: 32112072]. [PubMed Central: PMC7108143].

7. Wang D, Ju XL, Xie F, Lu Y, Li FY, Huang HH, et al. [Clinical analysis of 31 cases of 2019 novel coronavirus infection in children from six provinces (autonomous region) of northern China]. Zhonghua
Er Ke Za Zhi. 2020;58(4):269-74. Chinese. doi: 10.3760/cma.j.cn11214020200225-00138. [PubMed: 32118389].

8. Shen K, Yang Y, Wang T, Zhao D, Jiang Y, Jin R, et al. Diagnosis, treatment, and prevention of 2019 novel coronavirus infection in children: experts' consensus statement. World J Pediatr. 2020;16(3):223-31. doi: 10.1007/s12519-020-00343-7. [PubMed: 32034659]. [PubMed Central: PMC7090771].

9. Pediatric Branch of Hubei Medical Association; Pediatric Branch of Wuhan Medical Association; Pediatric Medical Quality Control Center of Hubei. [Recommendation for the diagnosis and treatment of novel coronavirus infection in children in Hubei (Trial version 1)]. Zhongguo Dang Dai Er Ke Za Zhi. 2020;22(2):96-9. Chinese. [PubMed: 32051073]. [PubMed Central: PMC7390014].

10. Li ZZ, Shen KL, Wei XM, Wang HL, Lu J, Tian H, et al. [Clinical analysis of pediatric SARS cases in Beijing]. Zhonghua Er Ke Za Zhi. 2003;41(8):5747. Chinese. [PubMed: 14744375].

11. Thabet F, Chehab M, Bafaqih H, Al Mohaimeed S. Middle East respiratory syndrome coronavirus in children. Saudi Med J. 2015;36(4):484-6. doi: 10.15537/smj.2015.4.10243. [PubMed: 25828287]. [PubMed Central: PMC4404484].

12. Hamilton KA, Hamilton MT, Johnson W, Jjemba P, Bukhari Z, LeChevallier $\mathrm{M}$, et al. Health risks from exposure to Legionella in reclaimed water aerosols: Toilet flushing, spray irrigation, and cooling towers. Water Res. 2018;134:261-79. doi: 10.1016/j.watres.2017.12.022. [PubMed: 29428779].

13. Zu ZY, Jiang MD, Xu PP, Chen W, Ni QQ, Lu GM, et al. Coronavirus Disease 2019 (COVID-19): A Perspective from China. Radiology. 2020;296(2):E15-25. doi: 10.1148/radiol.2020200490. [PubMed: 32083985]. [PubMed Central: PMC7233368].

14. Chen N, Zhou M, Dong X, Qu J, Gong F, Han Y, et al. Epidemiological and clinical characteristics of 99 cases of 2019 novel coronavirus pneumonia in Wuhan, China: a descriptive study. The Lancet. 2020;395(10223):507-13. doi: 10.1016/s0140-6736(20)30211-7.

15. Chung M, Bernheim A, Mei X, Zhang N, Huang M, Zeng X, et al. CT Imaging Features of 2019 Novel Coronavirus (2019-nCoV). Radiology. 2020;295(1):202-7. doi: 10.1148/radiol.2020200230. [PubMed: 32017661]. [PubMed Central: PMC7194022].

16. Song F, Shi N, Shan F, Zhang Z, Shen J, Lu H, et al. Emerging 2019 Novel Coronavirus (2019-nCoV) Pneumonia. Radiology. 2020;295(1):210-7. doi: 10.1148/radiol.2020200274. [PubMed: 32027573]. [PubMed Central: PMC7233366].

17. Wang Z, Chen X, Lu Y, Chen F, Zhang W. Clinical characteristics and therapeutic procedure for four cases with 2019 novel coronavirus pneumonia receiving combined Chinese and Western medicine treatment. Biosci Trends. 2020;14(1):64-8. doi: 10.5582/bst.2020.01030. [PubMed: 32037389]. 